\title{
An Investigative Analysis on Finding Patterns in Co-Author and Co-Institution Networks for LIDAR Research
}

\author{
Imran Ashraf, Soojung Hur, and Yongwan Park \\ Department of Information and Communication Engineering, Yeungnam University, South Korea
}

\begin{abstract}
Social Network Analysis (SNA) has proven itself to embody the complex relationships between actors of groups inside out. Not only that, but it has also emerged as a new paradigm to investigate the structure of ties and its role on relationships between the actors. This research aims to investigate the patterns of relationships between authors and institutions working in LIght Detection And Ranging (LIDAR) research area. LIDAR has been in the limelight during recent years, especially autonomous vehicles for map-making and objection detection tasks. Researchers need insight into the current contributors and research areas to devise policies and set future targets for this important technology. Current study performs SNA to identify potential institutions and researchers that can help to achieve those goals. National and international coauthorship is analysed separately. A total of 4274 papers from Web of Science (WOS) database are collected from 1998 to September 2017. SNA measures of degree, closeness, betweenness, and eigenvector centrality along with descriptive analysis are employed to study the patterns. Analysis reveals that the United States of America (USA) is the most central and significant country in terms of international co-authorship. China, Germany, the United Kingdom (UK) and Canada are ranked $2^{\text {nd }}$, $3^{r d}$, $4^{\text {th }}$ and $5^{\text {th }}$ in this list respectively. For co-institution network, National Aeronautics and Space Administration (NASA), University of Idaho and California Institute of Technology USA occupy $1^{\text {st }}, 2^{\text {nd }}$, and $5^{\text {th }}$ position respectively when top 5 institutions are considered. Consiglio NazionaleDelle Ricerche of Italy occupies $3^{\text {rd }}$ position while Chinese Academy of Science, China, secures $4^{\text {th }}$ place concerning betweenness centrality. Descriptive analysis reveals that during the last decade, co-author collaboration in scientific research has been elevated. Results show that research articles with 6 or more authors have higher citations than those with two to five authors. In addition, journals producing a higher number of papers and their corresponding citations are also discussed.
\end{abstract}

Keywords: Social network analysis, co-institution, co-authorship, LIDAR, degree, closeness, Eigenvector.

Received January 1, 2019; accepted April 8, 2020

https://doi.org/10.34028/iajit/17/6/6

\section{Introduction}

Although Social Network Analysis (SNA) has a considerable growth in the 1970s, yet during the last decade it has experienced a spark due to the proliferation of the increased number of social websites like Facebook, Twitter, and Instagram, etc. SNA has been linked to the theory of social capital recently [20], which states that Social Networks (SN) is a form of social capital which individuals can use to accelerate and advance their skills and opportunities. So, besides modern sociology, SNA has made its way to other fields of study including geography, communications studies, frauds and conflict analysis and information sciences, etc., SNA takes into account $\mathrm{SN}$ to investigate and interpret the ties between the actors of a network. In the beginning, the term SN was used in a metaphorical sense, until Barnes took the initiative to apply it analytically in the early 1950s. An $\mathrm{SN}$ is formed by individuals connected by different types of connections or ties. SNA focuses on analysing these relationships and flows betweenactors of the network to find out the structural patterns of the network. SNA also helps to determine the shape of the network and its suitability and benefits to its actors. For example, open networks possess higher importance as they are marked by a higher probability of new ideas and increased collaboration than those of closed networks.

In the modern globalized world of mutual cooperation, advent technologies and innovative research ideas are seen as an output of the wide and manifold collaboration. International collaboration is considered a trademark of excellence in quality of knowledge and the latest research. It not only helps to investigate and develop contemporary methodologies but also leads to disseminate knowledge to partner developing countries [9]. Such collaboration provides a common platform for generating and developing new tools and technologies globally [11]. Recent international collaborations have accelerated the coauthorship work which leads to the higher quality of standardization and enhances the visibility of the research [10].

SNA helps to execute performance analysis on such collaborative networks. The purpose of such analysis is 
to determine the direction of the research as well as the improvements and future planning. SNA indicators measured during the analysis help determine the weaknesses and strengths of the individuals and groups alike. Organizations and governments use these indicators to find suitable and leading individuals and groups working in a specific field. The research indicators also provide directions for governments to take initiatives and invest in the areas which lack research.

The rest of the paper is organized as follows. Section 2 describes a few research works related to the current study. Section 3 is about the data gathering process and adopted methodology. Section 4 discusses the results of the analysis. In the end, the conclusion is given.

\section{Related Work}

The co-authorship and co-institutional publications are the two most important units of collaboration. Since these provide the largest data about the networks of researchers, and recently many researchers used this data for analysis [7, 14, 18]. The Mehmood et al. [17] make use of SNA to investigate the social patterns in the emerging research area of Internet of Things (IoT). They use degree, betweenness, flow betweenness and eigenvector centrality are used for the analysis. Using SNA, Mehmood et al. [17] can determine the most central and pressure exerting countries in co-institution and co-author network. Research [17] identifies China as the most effective andleading country in IoT research. The Heng et al. [12] perform SNA on the coauthorship network for Dye-Sensitized Solar Cells (DSSCs) related Science Citation Index Expanded (SCIE) papers. The analysis aims at demonstrating the national and international cooperation of authors in the DSSC field. Their research concludes that the DSSC field has extensive cooperation at the national level in China, however, it lacks cooperation when the international level is concerned.

The Liu et al. [16] investigate co-authorship networks of the digital library research community for Association for Computing Machinery (ACM), Institute of Electrical and Electronics Engineers (IEEE) and joint ACM/IEEE papers. A new indicator Author Rank to find the impact of an individual author on the network is also proposed. Their analysis results show that PageRank and Author Rank are more advantageous analysis measures than those of degree centrality, closeness and between centrality. The Acedo et al. [2] perform SNA on the co-authorship network in management and organizational studies. Their research focuses on finding the trends in management and organizational studies and central authors within the research field. Results of the study show that there is a growing trend of co-authored papers in the specified field. With the help of SNA, they are also able to find the existing links between coauthors as well as the most central and influential authors within the research field.

The Abbasi et al. [1] investigate the effects of coauthorship on the performance of scholars in the field of Information Systems (IS). SNA measures including normalized degree, closeness, betweenness, eigenvector centrality and average ties strength and efficiency are used toevaluate the performance. Results of the research show that the scholars who have more connections to other scholars get a better citation index. Moreover, authors who have large ties strength exhibit better performance as compared to those with low tie strength. The Glänzel and Schubert [10] analyse social networks of co-authored Science Citation Index (SCI) papers from 1980 to 2000. Results of the study prove that the co-authorship not only is cost-effective but promotes the research activity as well. Similarly, the co-authorship network leads to higher productivity and a positive impact on innovation and development. The Koseoglu [15] explore the impact of co-authorship papers published in strategic management journal from 1980 to 2014 using descriptive analysis and SNA. It is pointed out that not only the number of institutions has increased but new institutions have also emerged in the domain during this period. In addition, the network of international collaboration is not based on geographical proximity, rather it is formed on international trade and social factors.

The Cheong and Corbitt [6] perform SNA on the coauthorship network of the pacific asia conference on information systems for the period of 1993 to 2008 . Sociogram of co-authorship network is provided using a directed network approach. SNA measures of degree, betweenness, closeness and eigenvector centrality, as well as structural hole, are used for analysis. Research affirms that co-authored papers have constantly been growing since 1993 and currently constitute $80 \%$ of the total papers. The Hou et al. [13] use SNA, cooccurrence analysis and frequency analysis of coauthorship network in Scientometrics journal. The study finds out the connected and unconnected authors of the journal. Individual authors with the highest degree, betweenness and closeness are also identified. Density, degree and betweenness centrality values of the whole network are very low suggesting that the network is not strongly connected and the collaborative network is very loose.

In this paper, we investigate the trend and structural patterns of co-authorship and co-institutional network for "LIght Detection And Ranging (LIDAR) technology" research field. LIDAR has been used since the 1960s, in many fields including urban planning, telecommunications, security services, forestry and recently in vehicles. The number of research papers is substantially increasing in this field, especially, after the Defence Advanced Research Projects Agency 
(DARPA) challenge in 2005 [4]. Usmani and Daud [23] propose a method to rank authors of a particular domain For this purpose, the popularity of the venue is taken into account to approximate the expected citations of the published work.

This study aims at analysing on how the co-author and co-institution network of this field has emerged and evolved over time. This paper aims at analysing the research work in LIDAR research area and identifies the potential research areas that need attention. Similarly, the leading institutions in the field are determined which helps new researchers to discuss novel and potential research ideas with the leading researchers. Struggling individuals and institutions can be supported to re-define policies and set new objectives to support research in LIDAR technology.

\section{The Research Method}

\subsection{Data Gathering}

Data for the analysis is collected from Thomson Reuters' Web of Science (WOS) database. This database includes editorials, chronologies, abstracts, proceedings of both journals and books and technical papers from distinguished and leading international journals. A short report published in February 2017 states that WOS has approximately 108.8 million records in total [21]. WOS data has authors address details as well as their institutional affiliations which are used to attribute the papers to concerning institutions and countries.

The data is collected for a period of 19 years from 1998 to September 2017. First, the search query "LIDAR" is executed to search the records. It results in 16,539 records in total. Web of Sciencehas 100 categories for LIDAR research area. We aim to collect only those papers which cover the usage of LIDAR in the transportation area covering ground vehicles only. So, we select only those categories which have relevant papers like "automation control system", "transportation", "robotics", etc., A total of 14 categories are selected which return 4756 records. These results include 4720 English, 11 Spanish, 9 German, 7 Portuguese, 4 Italian, 4 French and 1 paper written in the Korean language. Papers that are written in the English language only are considered. These records are sorted manually to exclude incomplete and irrelevant records. There on, 343 records are removed because of irrelevancy and 103 are rejected due to the missing data. Ultimately, a total of 4274 papers are selected for the final analysis

Algorithm 1: Finding records with national \& international coauthorship

for $k \leftarrow 1$ to $R$ do

Let $\mathbb{C}$ be the sorted array of countries of authors

for selected $k$

for $l \leftarrow 1$ to $\mathbb{C} d o$

if $\mathbb{C}$ for selected $k$ is not the same then

$$
\begin{aligned}
& \quad \text { break } \\
& \quad R_{I A}=R_{k} \\
& R_{N A}=R_{k} \\
& \text { break } \\
& \text { end if } \\
& \text { end for } \\
& \text { end for } \\
& \text { return vector }\left\{R_{I A}, R_{N A}\right\}
\end{aligned}
$$

The primary aim of this research is to investigate international co-authorship and co-institution network. Since papers from WOS include both national and international papers, we need to select only those papers whose authors' affiliations are from different countries. Algorithm 1 is designed for the said purpose. The implementation of the algorithm is done using Matlab R2015b. Algorithm 1 takes the collected records $R$ as input and returns two vectors $\mathrm{R}_{\mathrm{IA}}$ and $\mathrm{R}_{\mathrm{NA}}$. $\mathrm{R}_{\mathrm{IA}}$ contains the records of the papers written by coauthors where at least one of them is from a different country. Whereas the papers written by the authors of the same country are in $\mathrm{R}_{\mathrm{NA}}$. Author's current institutional affiliation is used to categorize the records into the national and international authorship category. These vectors are later used for national and international co-authorship analysis. During its execution, Algorithm 1 accesses the affiliated countries of authors of a paper and they are added to $\mathrm{R}_{\mathrm{NA}}$ if the countries are same, otherwise to $\mathrm{R}_{\mathrm{IA}}$.

\subsection{Analysis Tools and Accuracy Measures}

The analysis is performed using a variety of tools including SNA tools and custom-defined functions in Matlab. The analysis is focused on the network of authors and institutions that work together in groups. So, the basic entity for the analysis is the link between authors and institutions. The nature of this connection is analysed with SNA tools like Pajek, Bib excel and UCINet, etc.

UCINet is one of the many analytical tools available for SNA [22] and is used quite frequently for such analysis. First, an adjacency matrix from the records gathered from WOS is prepared and then this matrix is used to make symmetrical networks for co-authorship and co-institution. Besides, the WOS record file is also processed with Bib excel [3] and graphs are designed using Pajek software [19]. Later on, SNA is used to measure the structural patterns in these networks. A wide variety of measures like centralization, density, effectiveness etc., can be used for this purpose. The following section describes the SNA measures used for the analysis in this paper. These measures are the indicators which describe the cohesion of a network and role of an actor in the network.

\subsubsection{Degree Centrality}

Degree centrality can be defined as the number of links incident upon an actor/node in a network [8]. 
Mathematically, degree centrality $d$ (i) for node $i$ is written as:

$$
d(i)=\sum_{i} m_{i j}
$$

Where,

$$
m_{i j}= \begin{cases}1, & \text { if there is a link } \\ 0, & \text { if there is no link }\end{cases}
$$

Degree centrality is the centrality of a network from 'degree' perspective, which implies in and outflows from each actor as a centre. It can also be computed without direction consideration of ties; a tie in either direction is considered as a tie. Degree centrality denotes the active player in a network who serves as a hub.

\subsubsection{Closeness Centrality}

Closeness centrality of an actor in a network is the total distance of that actor from all other actors. In the mathematical formula, it can be written as:

$$
c(i)=\sum_{i} d_{i j}
$$

Where $d_{i j}$ is the number of links from actor $i$ to $j$ and $c(i)$ is the closeness of actor $i$. Closeness is an inverse metric which means that large value actor is less central in the network. So, often, normalized closeness is used for analysis; Freeman normalized closeness is used for analysis in this study.

\subsubsection{Betweenness Centrality}

Between centrality can be defined as the time that an actor $i$ needs to reach actor $k$ using actor $k$ using the shortest path [8]. In other words, it is the number of shortest paths that go through a given actor. Mathematically, it can be written as:

$$
b(i)=\sum_{j, k} \frac{g_{j i k}}{g_{j k}}, i \neq j \neq k
$$

Where, $g_{j k}$ is the number of shortest paths from actor $j$ to $k$ and $g_{j k}$ is the number of shortest paths for the same actors through $i$. Betweenness centrality identifies an actor's position who acts as a single point of failure. The purpose of betweenness centrality is to identify how much extent an actor facilitates the flow of a network.

\subsubsection{Eigenvector Centrality}

Eigenvector centrality is another popular measure used in SNA by [5]. It is the principal eigenvector calculated based on the adjacency matrix of a network. Its equation is:

$$
\lambda v=A v
$$

Where $A$ represents the adjacency matrix of the graph, $\lambda$ is the eigenvalue which is a constant, and $v$ is the eigenvector. Eigenvector centrality shows the influence of an actor in a network. It shows how close an actor is to other high close actors. It implies that an actor is important if other important actors are connected to it.
If differs from betweenness centrality; an actor with more links does not necessarily award high eigenvector centrality. Similarly, an actor with high eigenvector does not imply that it has a higher number of links as, an actor may have few but very important links.

\section{Results and Discussions}

\subsection{International Co-Authorship Network}

Using the publication data filtered with Algorithm 1, the adjacency matrix for the co-authorship network is formulated. This matrix is then used in UCINet to calculate the SNA indicators. Table 1 shows the results for four indicators for the top 20 countries.

\subsubsection{Degree, Closeness and Eigenvector Centrality}

The USA, China and Germany are the most central countries as given in Table 1 , in terms of degree centrality for the LIDAR research area. The USA has the highest number of co-authored papers with other countries. The degree centrality value of USA is 591, which means that the USA accounts for $22.45 \%$ of the whole network concerning degree centrality. It is followed by China, Germany, UK, Canada, Australia, France, Italy, Spain and Netherlands in the given order. Apart from China, three other Asian countries including Japan, South Korea and India are also able to secure a place among the top 20 countries.

Table 1 shows the list of top 20 countries for their nodal degree, closeness, betweenness and eigenvector centrality. Spain, Netherlands, Norway, Sweden, Finland and Japan perform better concerning betweenness, closeness and eigenvector centralities and improve their positions in the list. China is unable to maintain its positions in closeness and betweenness centralities and thus moves to $7^{\text {th }}$ and $10^{\text {th }}$ position in closeness and betweenness centralities, respectively. The USA holds the first position in all centrality measures. It is also worth mentioning that Italy, Denmark, India and Greece manage to secure a position in the top 20 list concerning closeness.

Table 1. Structural-hole values by the country for co-authorship network.

\begin{tabular}{|c|c|c|}
\hline Rank & Country & Effect \\
\hline 1 & USA & 44.50 \\
\hline 2 & UK & 31.98 \\
\hline 3 & Germany & 29.90 \\
\hline 4 & Netherlands & 28.71 \\
\hline 5 & France & 24.17 \\
\hline 6 & Australia & 22.76 \\
\hline 7 & Spain & 21.74 \\
\hline 8 & Sweden & 18.72 \\
\hline 9 & Italy & 18.72 \\
\hline 10 & China & 18.33 \\
\hline 11 & Canada & 18.12 \\
\hline 12 & Finland & 17.73 \\
\hline 13 & Norway & 16.99 \\
\hline 14 & Japan & 16.15 \\
\hline 15 & Austria & 14.72 \\
\hline 16 & Scotland & 14.51 \\
\hline 17 & Switzerland & 13.96 \\
\hline 18 & Denmark & 12.57 \\
\hline 19 & Greece & 12.33 \\
\hline 20 & Wales & 10.53 \\
\hline
\end{tabular}


Figure 1 indicates the picture for international coauthorship network. The network consists of only those countries who got at least 15 co-authored papers as displaying all the 85 countries is not appropriate due to space and picture quality limitations. Co-authorship network analysis reveals that many countries with higher centrality values are connected to the USA, China and the UK. The USA has very strong ties with European countries as well as China, South Korea and
South Africa. The UK has smooth ties with neighbouring countries in addition to Australia. China is closely associated withthe USA, Canada and the Netherlands. Lines connecting the countries represent that the countries are connected as they co-authored papers. The thickness of lines in the network is based on the number of co-authored papers. The number of co-authored papers is also shown with each line.

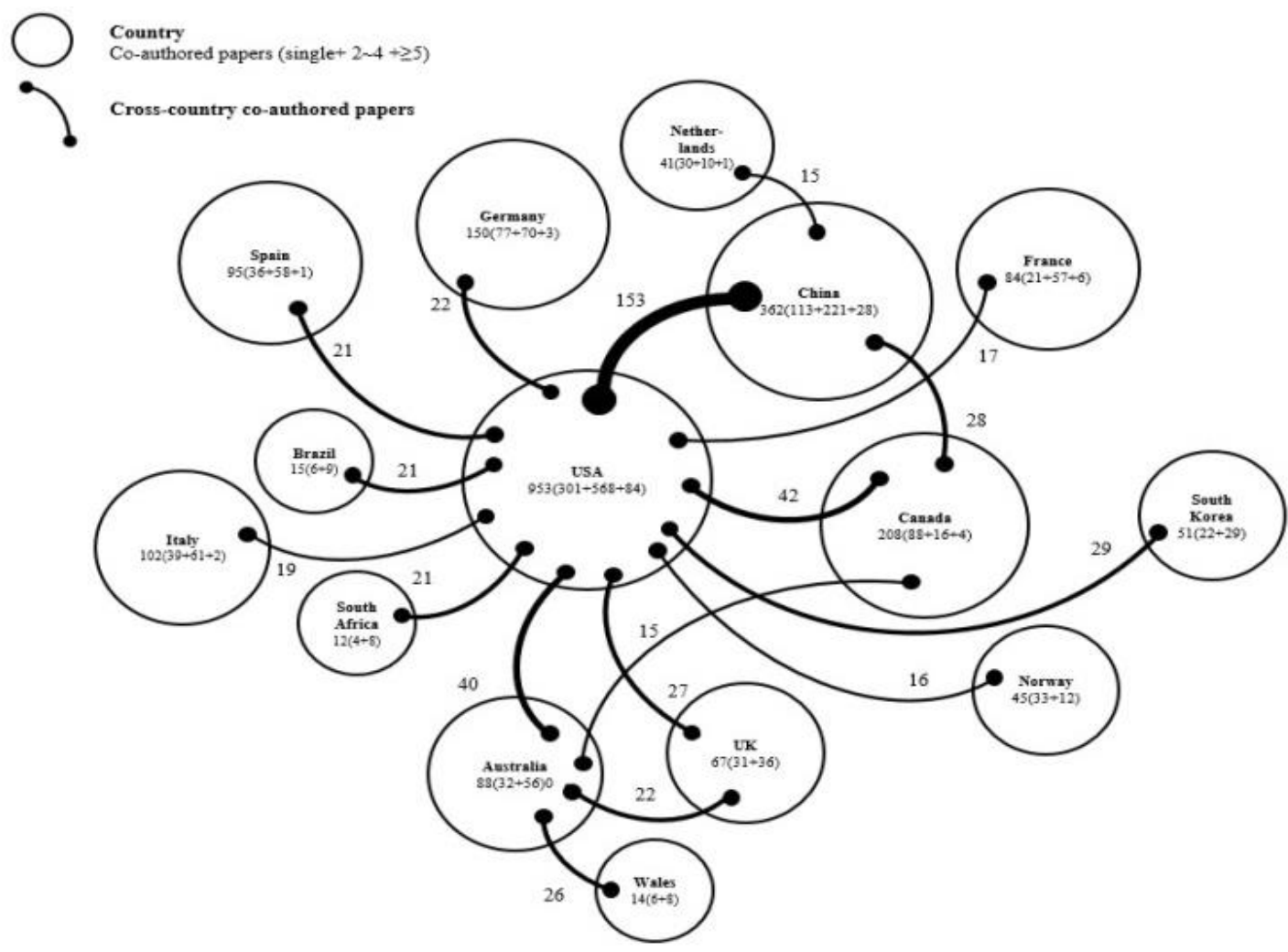

Figure 1. National and International co-authorship network for LIDAR research.

Analysis reveals that the cross country collaboration mostly takes place in connection with the USA as the majority of 85 countries are connected to the USA. This is primarily owing to the research projects being worked on in USA universities and research institutions. So, under-developed and developing countries benefit from the latest research projects by networking with USA institutions. Moreover, China, UK, Australia and Canada also hold central positions and work as a hub for other nodes. It is also important to mention that the countries that have at least 15 coauthored papers are shown in the network map for the sake of simplicity. In addition, the number of intracountry published papers are shown inside the circles. Intra-country published papers are further categorized into a single author, 2-4 authors and $\geq 5$ authors' papers. The analysis of intra-country papers shows that the number of multi-authored papers is higher than those of single-author papers which is a clear indication that during the recent years the trend of coauthorship even at country level has been elevated. The only exceptions are Netherlands and Norway where single-author papers are higher than multi-author papers.

Table 2 depicts the effectiveness values of the 'structural holes'. Structural holes are indicators which show the strategic management of countries in terms of co-authorship and co-institution networks. According to Burt [5], structural holes generate a competitive advantage for countries whose networks span the holes. Structural holes take the potential to work as brokers to control the flow of information between nodes and control the co-works that bring the nodes together from opposite sides of holes. Table 2 displays the effectiveness of the countries in terms of structural holes. It is notable that in terms of top 20 countries concerning their degree, closeness, betweenness and eigenvector centrality, occupy the central positions in this table as well. 
Table 2 . Centrality values by country.

\begin{tabular}{|c|c|c|c|c|c|c|c|c|}
\hline Rank & Country & Degree & Country & Closeness & Country & Betweenness & Country & Eigenvector \\
\hline 1 & USA & 591 & USA & 0.555 & USA & 1366.871 & USA & 0.637 \\
\hline 2 & China & 299 & UK & 0.526 & UK & 741.083 & China & 0.557 \\
\hline 3 & Germany & 194 & Germany & 0.509 & Netherlands & 512.919 & Canada & 0.270 \\
\hline 4 & UK & 187 & Spain & 0.488 & Germany & 402.582 & Australia & 0.209 \\
\hline 5 & Canada & 173 & Netherlands & 0.485 & Australia & 335.561 & Germany & 0.170 \\
\hline 6 & Australia & 165 & Canada & 0.482 & Spain & 231.094 & UK & 0.167 \\
\hline 7 & France & 118 & China & 0.482 & Italy & 191.367 & South Korea & 0.120 \\
\hline 8 & Italy & 112 & Australia & 0.476 & France & 183.149 & Spain & 0.108 \\
\hline 9 & Spain & 109 & Norway & 0.476 & Austria & 162.754 & France & 0.104 \\
\hline 10 & Netherlands & 88 & Sweden & 0.476 & China & 152.701 & Italy & 0.104 \\
\hline 11 & Norway & 79 & Finland & 0.471 & Norway & 126.958 & Brazil & 0.088 \\
\hline 12 & Sweden & 69 & Italy & 0.468 & Finland & 118.761 & Netherlands & 0.083 \\
\hline 13 & Wales & 69 & France & 0.466 & Greece & 114.714 & Norway & 0.080 \\
\hline 14 & Finland & 66 & Denmark & 0.450 & Scotland & 114.357 & Wales & 0.074 \\
\hline 15 & Switzerland & 61 & Japan & 0.448 & Canada & 113.870 & Finland & 0.073 \\
\hline 16 & South Korea & 56 & Switzerland & 0.445 & Japan & 103.181 & South Africa & 0.070 \\
\hline 17 & Austria & 54 & India & 0.438 & Ireland & 102.341 & Sweden & 0.065 \\
\hline 18 & Japan & 51 & Brazil & 0.435 & Sweden & 85.265 & Switzerland & 0.057 \\
\hline 19 & Brazil & 50 & Austria & 0.429 & South Korea & 76.278 & Japan & 0.056 \\
\hline 20 & Portugal & 42 & Greece & 0.429 & Switzerland & 74.398 & Portugal & 0.048 \\
\hline
\end{tabular}

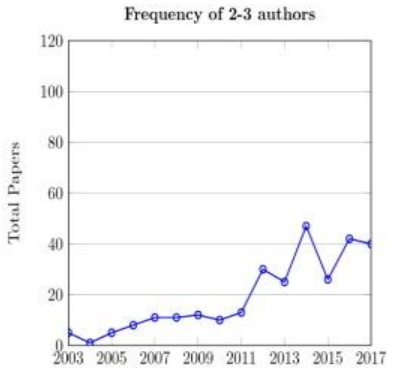

a) Papers published by $2-3$ authors.

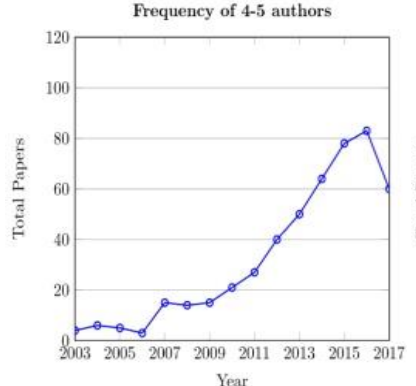

b) Papers published by $4-5$ authors.

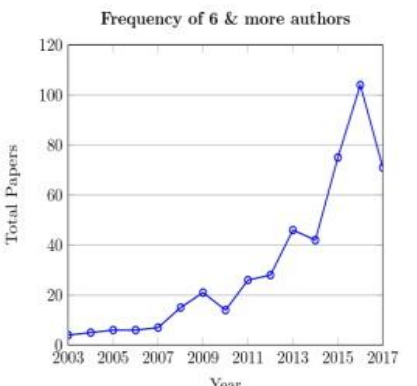

c) Papers published by $6 \&$ more authors.

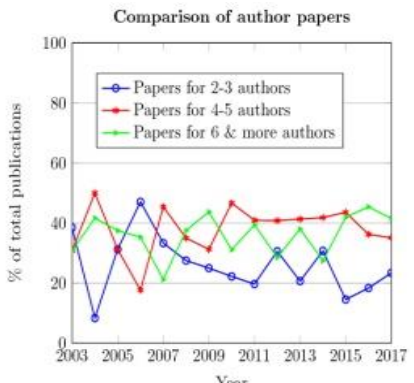

d) Comparison of authors' papers.

Figure 2. Frequency of published papers for authors.

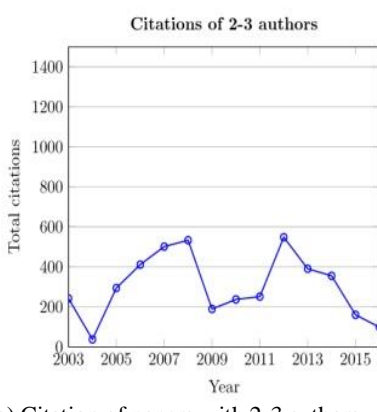

a) Citation of papers with 2-3 authors.

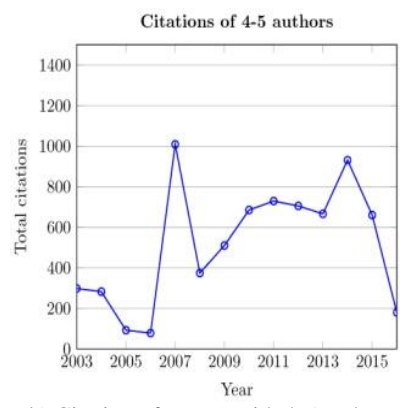

b) Citation of papers with 4-5 authors.
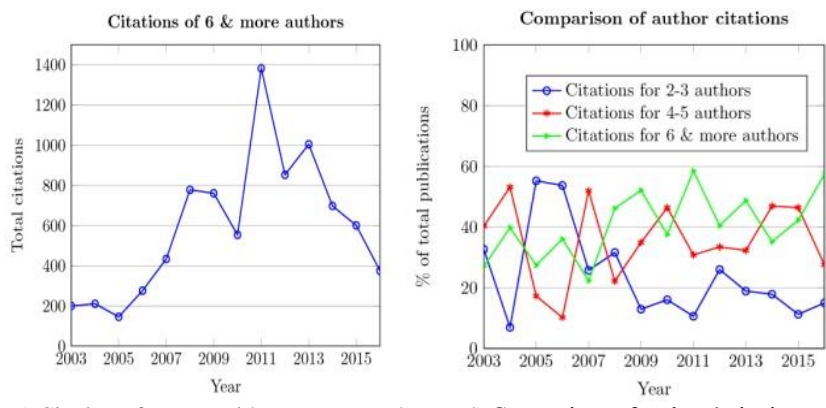

Figure 3. Citations for papers 2003 to 2016.

\subsubsection{Percentage of $2-3,4-5$ and $\geq 6$ Authors}

We make a comparison of 2-3 author, 4-5 author and $\geq 6$ author papers in LIDAR research area for the period of 2003 to 2017. Figure 2 shows the results for the analysis. It is evident that the number of multipleauthor papers has been increased over the period. However, the graph showing number of papers for 4-5 authors is more stable in comparison. Similarly, papers written by $4-5$ authors have highest publications. Also, the ratio of papers for 4-5 authors to the total publications is stable as well. The graph shows a reduced number of papers for 2017. The reason for this reduction is that the papers have been selected until September 2017 only. So, exclusion of the last three and half month of 2017 results in a reduced number of papers.

Figure 3 shows a comparison between 2-3 author and $\geq 6$ author papers. It reveals that authors tend to work in a group in the LIDAR research area has been increased over the period and resulted in a higher number of publications.It shows the evolution of coauthors network over time and demonstrates that the co-author network is expanding day by day. The primary reason for such expansion is the reward which is an increased number of papers and citations when authored by a higher number of authors. Working in a group are followed by joint discussions that result in innovative and novel ideas and are more productive. Moreover, junior researchers can benefit from the expertise of senior researchers that also improve their performance.

\subsubsection{Citations for $2-3,4-5$, and $\geq 6$ Authors}

An analysis to study the impact of co-authorship on the number of citations is also made here. Figure 4 shows the results of the analysis. It shows that citations have 
observed ups and downs during the years as some good papers can get a higher number of citations. However, ratios of the citation graph show that papers written by $\geq 6$ authors got the highest number of citations. It is also evident that the graphs for paper citations are not very stable.

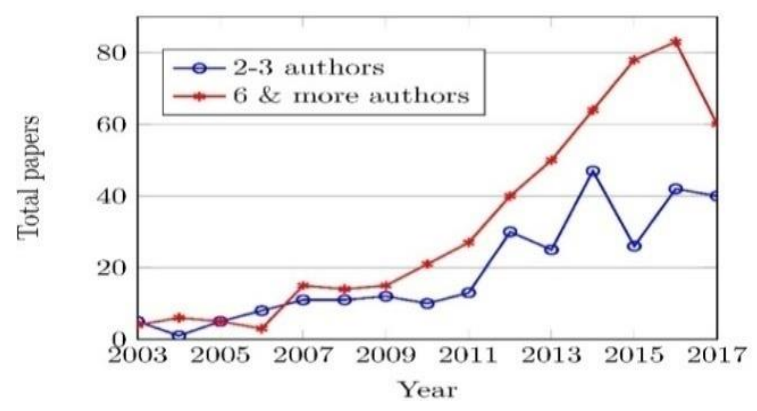

Figure 4. Publications for $2-3$ vs $\geq 6$ authors 2003 to 2016 .

Increase in citations of papers with a higher number of authors may be linked to several factors. First, a higher number of authors indicate a large network that helps to get more citations. Second, citations also depend upon the choice of journals in which a paper is published. Open access journal papers are free to download that increases the probability of being read by more researchers than that of non-open access journals.

\subsection{International Co-institutional Network}

Similar to co-authorship network analysis, a separate adjacency matrix is formulated for the international coinstitutional network using the publication data. UCINet is then used on the adjacency matrix to calculate the SNA measures of degree, betweenness and eigenvector centrality. A total of 1134 institutions are selected while making the adjacency matrix.

\subsubsection{Degree, Betweenness and Eigenvector Centrality}

Table 3 summarizes the results for the international coinstitutional network. Results reveal that National Aeronautics and Space Administration (NASA) has the highest degree centrality and secures 1 st place on the list. University of Idaho, USA, has the 2nd position in degree centrality list with a minimal difference from Consiglio Nazionale Delle Ricerche, Italy, who occupies 3rd position in degree centrality list. Chinese Academy of Science possesses 4th position followed by California Institute of Technology USA, National Resources Canada, CSIRO Marine and Atmospheric Research Australia, Wuhan University China, University of Maryland USA and Norwegian University of Life Sciences Norway in the given order. NASA possesses the same position in betweenness centrality list, whereas 2nd position is now occupied by Norwegian University of Life Sciences and 3rd by US Forest Service. Few institutions which were not part of top 20 list of degree centrality performed better and now occupy central positions in betweenness centrality list. Among these institutions are Science Systems and Applications, Inc. and University of New South Wales from the USA, University of Cambridge and the University of Leeds from the UK, the University of Tehran from Iran, Technical University of Denmark and the University of Cologne, Technical University Munchen and Wageningen University from Germany. Considering only the top 20 institutions, USA holds $40 \%$ and $20 \%$ of the network in terms of degree and betweenness centrality. Similarly, China accounts for $15 \%$ and $10 \%$ of the network for degree and betweenness centrality respectively.

Results for eigenvector centrality indicate that USA institutions hold the most central and influential positions. The USA accounts for $30 \backslash \%$ of the whole network, with China and Australia keeping 20\\% each. No institution from the UK is listed in the top 20 list of eigenvector centrality. As we know that eigenvector does not consider the number of ties among nodes like degree centrality, rather it ranks the nodes higher which are between other highly close nodes. In this way, preference is given to the nodes which are more important and central. In the current analysis, USA institutions are more important and central in LIDAR research area when eigenvector centrality is considered.

\subsubsection{Citation Analysis}

The citation analysis is mapped using Pajek and results are shown in Figure 5. Papers that got at least 40 citations and got co-citations as well are depicted in the figure. Each circle corresponds to a paper while node colour denotes the publication year of the paper. Line width indicates the number of citations a paper has received.

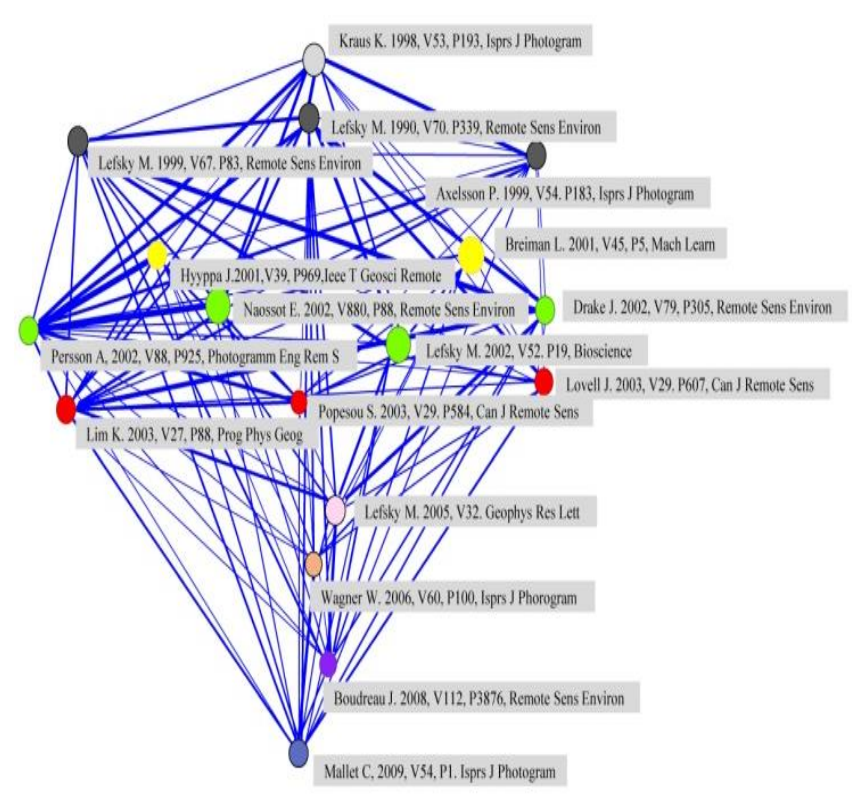

Figure 5. Citation network. 
Table 3. Centrality values for institutions in co-institutional network.

\begin{tabular}{|c|c|c|c|c|c|c|}
\hline Rank & Institution & Degree & Institution & Betweenness & Institution & Centrality \\
\hline 1 & $\begin{array}{c}\text { California Institute of } \\
\text { Technology }\end{array}$ & 45 & $\begin{array}{c}\text { California Institute of } \\
\text { Technology }\end{array}$ & 33480.59 & University of Waterloo & 0.664 \\
\hline 2 & Carnegie Institution for Science & 42 & Wuhan University, China & 23764.16 & Xiamen University, China & 0.640 \\
\hline 3 & University of Leeds & 35 & University of California & 20588.76 & Wuhan University, China & 0.202 \\
\hline 4 & University of New Hampshire & 35 & NASA & 20030.49 & $\begin{array}{c}\text { Huaiyin Institute of } \\
\text { Technology }\end{array}$ & 0.171 \\
\hline 5 & United States Geological Survey & 30 & University of Maryland & 19845.72 & $\begin{array}{c}\text { Nanjing Univ. of Sci. \& } \\
\text { Technology }\end{array}$ & 0.171 \\
\hline 6 & Wuhan University, China & 29 & Carnegie Institution for Science & 19775.72 & $\begin{array}{l}\text { National Univ. of Defence } \\
\text { Tech. }\end{array}$ & 0.130 \\
\hline 7 & NASA & 28 & Yonsei University & 17431.86 & Ryerson University, Canada & 0.084 \\
\hline 8 & University of Waterloo & 28 & Seoul National University & 17431.20 & $\begin{array}{l}\text { Changjiang Spatial Info. } \\
\text { Tech. Eng. }\end{array}$ & 0.080 \\
\hline 9 & Texas A \& M University & 26 & University of Tennessee & 17330.81 & $\begin{array}{c}\text { Chinese Academy of } \\
\text { Sciences }\end{array}$ & 0.053 \\
\hline 10 & United States Forest Service & 26 & Georgia Institute of Tech. & 16920.81 & University of Toronto & 0.052 \\
\hline 11 & Chinese Acad. Of Science & 25 & University of Cambridge & 16680.00 & $\begin{array}{l}\text { Wuhan University of } \\
\text { Technology }\end{array}$ & 0.041 \\
\hline 12 & University Of Oxford & 25 & Ohio State University & 15523.86 & Purdue University & 0.030 \\
\hline 13 & Lund University, Sweden & 24 & Oregon State University & 15201.38 & Shenzhen University & 0.028 \\
\hline 14 & University Of Maryland & 24 & Arizona State University & 12123.60 & Sun Yat-Sen University & 0.028 \\
\hline 15 & Xiamen University, China & 23 & University of Calgary & 12019.36 & University of Tennessee & 0.028 \\
\hline 16 & Curtin University, Malaysia & 22 & University of Oxford & 11703.47 & University of Wurzburg & 0.028 \\
\hline 17 & University Of Western Australia & 21 & University of Twente & 11508.11 & Nanchang University & 0.027 \\
\hline 18 & University Of Witwatersrand & 21 & George Mason University & 11457.56 & $\begin{array}{c}\text { Collab. Inn. Centre for Geos. } \\
\text { Tech. }\end{array}$ & 0.026 \\
\hline 19 & Brown University & 20 & Karlsruhe Institute of Tech. & 10612.28 & $\begin{array}{c}\text { Univ. of Elect. Sci. \& } \\
\text { Technology }\end{array}$ & 0.026 \\
\hline 20 & Embrapa Satellite Monitoring & 20 & US Forest Service & 9304.62 & George Mason University & 0.024 \\
\hline
\end{tabular}

\subsection{Journal Analysis}

In research, journals with a higher number of citations (impact factor) are preferred for publication. A separate analysis of journals who got higher citations is also performed in this regard. Figure 6 shows the results for the analysis for the top 10 journals with the highest number of citations in the field of LIDAR technology for the period of 2003 to 2016. It shows that remote sensing of environment journal of elsevier is very consistent in getting a higher number of citations for its published papers. Besides, it also got the highest citations for its papers from 2003 to 2016.

\section{Conclusions}

This research investigates the changes in structural patterns and network of researchers in the field of LIDAR technology research area. The research takes into account the co-authorship and co-institutional records, gathered from the web of science from 1998 to September, 2017. SNA is performed in order to evaluate the influence and control that different institutions, authors and journals possess in international co-authorship network. Results of analysis reveal that USA has the highest number of co-authored publications followed by China, Australia and Germany. Other countries in this list include UK, Canada, France, Italy, Spain and Netherlands in terms of structural hole and centrality measures.Network map for co-authorship shows that the USA, China and Australia hold the most influential and central positions based on their connections to other countries.

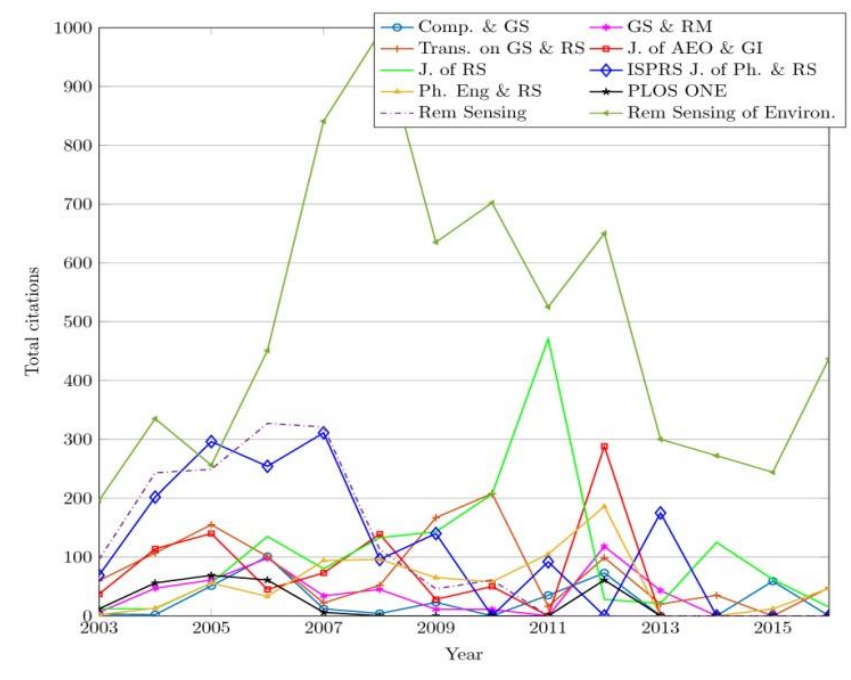

Figure 6. Citations for top 10 journals.

Besides China, other Asian countries including South Korea, Japan and India and one African country South Africa also hold central positions in coauthorship network. These countries made their way to top 20 list because they have very strong ties with the USA who holds the highest position in the list.

Results for co-authorship paper citations show that the tendency of researchers working in groups has been increased over time. Although papers produced by 2-3 authors and $\geq 6$ authors have been increased yet, papers written by $4-5$ authors have very consistent increase over the period. Citation data affirms that papers with $\geq 6$ authors tend to get a higher number of citations than other papers. Results show that the highest number of citations are for papers with 6 or more authors. Analysis for journals with high citations 
indicates that Remote Sensing of Environment Journal of Elsevier is very consistent in getting a higher number of citations for its published papers in LIDAR research area.

The results for co-institutional network analysis unfold that USA institutions control central positions in terms of degree, betweenness and eigenvector centrality. NASA holds the highest position followed by the University of Idaho and Consiglio Nazionale Delle Ricerche of Italy. It is also noteworthy to mention that four institutions from Australia including CSIRO Marine and Atmospheric Research, Curtin University, University of South Australia and the University of West Australia also hold influential positions in the coinstitutional network. For the top 20 institutions, USA occupies $40 \%, 25 \%$ and $30 \%$ of the network in terms of degree, betweenness and eigenvector centrality. China holds $10 \%$ and $20 \%$ for degree and betweenness while Australia accounts for $20 \%$ of the co-institutional network for eigenvector centrality.

To conclude, we can say that the USA holds the most dominant and influential positions for coauthorship network. While for the co-institutional network, USA and China both possess important and central positions. Future work is to include PageRank as a measure for analysis. A separate study considering Scopus and Google Scholar for analysing and investigating the difference in collaboration network is also under process. The current study does not consider the category of the publishing journal, i.e., open and non-open access, which might be an interesting factor to consider for citation analysis. Future work considers the impact of journal category on researchers' network as well.

\section{Financial Disclosure}

This research was supported by the MSIT (Ministry of Science and ICT), Korea, under the ITRC (Information Technology ResearchCenter) support program (IITP2020-2016-0-00313) supervised by the IITP (Institute for Information and communication Technology Promotion). This work was also supported by the 2019 Yeungnam University Research Grant

\section{References}

[1] Abbasi A., Altmann J., and Hossain L., "Identifying the Effects of Co-Authorship Networks on The Performance of Scholars: A Correlation and Regression Analysis of Performance Measures and Social Network Analysis Measures," Journal of Informetrics, vol. 5, no. 4, pp. 594-607, 2011.

[2] Acedo F., Barroso C., Casanueva C., and Galán J., "Co-Authorship in Management and Organizational Studies: An Empirical and
Network Analysis," Journal of Management Studies, vol. 43, no. 5, pp. 957-983, 2006.

[3] Bib excel Software. Available: http://homepage.univie.ac.at/juan.gorraiz/bibexc el/, Last Visited, 2020.

[4] Buehler M. Iagnemma K., and Singh S., The 2005 DARPA Grand Challenge: The Great Robot Race, Springer Science and Business Media, 2007.

[5] Burt R., "Structural Holes and Good Ideas," American Journal of Sociology, vol. 110, no. 2, pp. 349-399, 2004.

[6] Cheong F. and Corbitt B., "A Social Network Analysis of the Co-Authorship Network of The Pacific Asia Conference on Information Systems From 1993 To 2008," in Proceedinds of Pacific Asia Conference on Information Systems, Hyderabad, pp. 23, 2009.

[7] Cho C., Hu M., and Liu M., "Improvements in Productivity Based on Co-Authorship: A Case Study of Published Articles in China," Scientometrics, vol. 85, no. 2, pp. 463-470, 2010.

[8] Freeman L., "Centrality in Social Networks Conceptual Clarification," Social Networks, vol. 1, no. 3, pp. 215-239, 1978.

[9] Freshwater D., Sherwood G., and Drury V., "International Research Collaboration: Issues, Benefits and Challenges of the Global Network," Journal of Research in Nursing, vol. 11, no. 4, pp. 295-303, 2006.

[10] Glänzel W. and Schubert A., Handbook of Quantitative Science and Technology Research, Springer, 2004.

[11] Grossetti M., Eckert D., Gingras Y., Jégou L., Larivière V., and Milard B., "Cities and The Geographical Deconcentration of Scientific Activity: A Multilevel Analysis of Publications (1987-2007)," Urban Studies, vol. 51, no. 10, pp. 2219-2234, 2014.

[12] Heng X., Li R., Wang X., and Zhang Q., "Analysis on Co-Authorship Network: Applied to Dye-Sensitized Solar Cells," Chemical Engineering III, pp. 209, 2013.

[13] Hou H., Kretschmer H., and Liu Z., "The Structure of Scientific Collaboration Networks in Scientometrics," Scientometrics, vol. 75, no. 2, pp. 189-202, 2007.

[14] Inzelt A., Schubert A., and Schubert M., "Incremental Citation Impact Due to International Co-Authorship in Hungarian Higher Education Institutions," Scientometrics, vol. 78, no. 1, pp. 37-43, 2008.

[15] Koseoglu M., "Mapping the Institutional Collaboration Network of Strategic Management Research: 1980-2014," Scientometrics, vol. 109, no. 1, pp. 203-226, 2016. 
[16] Leclerc M. and Gagné J., "International Scientific Cooperation: The Continentalization of Science," Scientometrics, vol. 31, no. 3, pp. 261-292, 1994.

[17] Liu X., Bollen J., Nelson M., and Van de Sompel H., "Co-authorship Networks in The Digital Library Research Community," Information Processing and Management, vol. 41, no. 6, pp. 1462-1480, 2005.

[18] Mehmood A., Choi G., Feigenblatt O., and Park H., "Proving Ground for Social Network Analysis in the Emerging Research Area 'Internet of Things'(IoT),” Scientometrics, vol. 109, no. 1, pp. 185-201, 2016.

[19] Pajek Software. Available: http://valdo.fmf.unilj.si/pub/networks/pajek/, Last Visited, 2020.

[20] Putnam R., Culture and politics, Springer, 2000.

[21] Reuters T., 2017. Available: http://images.infro.science.thomsonreuters.biz/, Last Visited, 2020.

[22] UCINet Software. Available: http://sites.google.com.site.ucinetsoftware/home, Last Visited, 2020.

[23] Usmani A. and Daud A., "Unified Author Ranking based on Integrated Publication and Venue Rank," The International Arab Journal of Information Technology, vol. 14, no. 1, pp. 111117, 2017.

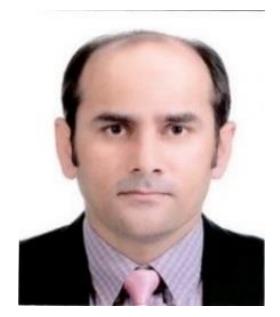

Imran Ashraf received his Ph.D. in Information \& Communication Engineering from Yeungnam University, South Korea in 2018 and the M.S. degree in computer science from the Blekinge Institute of Technology, Karlskrona, Sweden, in 2010. He has worked as a postdoctoral fellow at Yeungnam University, as well. Currently, he is working as an Assistant Professor atthe Information and Communication Engineering Department, Yeungnam University,Gyeongsan, South Korea. His research areas include indoor positioning and localization, indoor location based services in wireless communication, sensors (LIDAR) for smart car, and data mining.

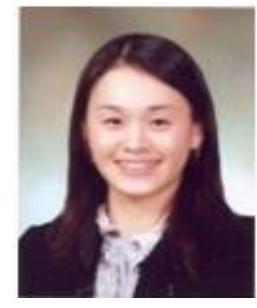

Soojung Hur received her B.S degree from Daegu University, Gyeongbuk, Korea in 2001. She finished M.S course in Electrical Engineering from San Diego State University of San Diego in 2004. And she received M.S and Ph. D degree in Information and Communication Engineering from Yeungnam University, Korea in 2007 and 2012 respectively. She is working as research professor at the Mobile Communication Laboratory, Yeungnam University. Her current research interests include performance of mobile communication, indoor/outdoor location and unmanned vehicle.

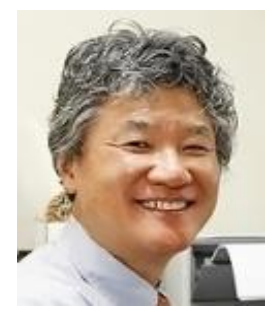

Yongwan Park received the $\mathrm{BE}$ and $\mathrm{ME}$ degrees in Electrical Engineering from Kyungpook University, Daegu Korea, in 1982 and 1984, respectively, and his MS and $\mathrm{PhD}$ degrees in Electrical Engineering from State University of New York at Buffalo, USA in 1989 and 1992, respectively. He worked at California Institute of Technology as a research fellow from 1992 to 1993. From 1994 to 1996, he served as a chief researcher for developing IMT-2000 system at SK Telecom, Korea. Since 1996, he has been a professor of information and communication engineering at Yeungnam University, Korea. From January to February of 2000, he was an invited professor at NTT DoCoMo Wireless Lab., Japan. He was also a visiting professor at UC Irvine, USA in 2003. From 2008 to 2009 he served as a director of Technology Innovation Center for wireless multimedia by Korean government. From 2009 to March 2017, he also served as the president of GITC (Gyeongbuk Institute of IT Convergence Industry Technology), Korea. He is also serving as the chairman of 5G Forum Convergence Service Committee in Korea. His current research areas of interest includes 5G systems in communication, OFDM, PAPR reduction, indoor location-based services in wireless communication and smart sensors (LIDAR) for smart car. 